\title{
Emerging cephalosporin and multidrug-resistant gonorrhoea in Europe
}

M J Cole (michelle.cole@phe.gov.uk)1, G Spiteri², S A Chisholm², S Hoffmann³, C A Ison'1, M Unemo4, M Van de Laar²

1. Public Health England, London, United Kingdom

2. European Centre for Disease Prevention and Control, Stockholm, Sweden

3. Statens Serum Institut, Copenhagen, Denmark

4. Örebro University Hospital, Örebro, Sweden

Cole MJ, Spiteri G, Chisholm SA, Hoffmann S, Ison CA, Unemo M, Van de Laar M. Emerging cephalosporin and multidrug-resistant gonorrhoea in Europe. Euro Surveill. 2014;19(45):pii=20955. Available online: http://www.eurosurveillance.org/ViewArticle.aspx?Articleld=20955

Neisseria gonorrhoeae has consistently developed resistance to antimicrobials used therapeutically for gonorrhoea and few antimicrobials remain for effective empiric first-line therapy. Since 2009 the European gonococcal antimicrobial surveillance programme (Euro-GASP) has been running as a sentinel surveillance system across Member States of the European Union (EU) and European Economic Area (EEA) to monitor antimicrobial susceptibility in $N$. gonorrhoeae. During 2011, N. gonorrhoeae isolates were collected from 21 participating countries, and $7.6 \%$ and $0.5 \%$ of the examined gonococcal isolates had in vitro resistance to cefixime and ceftriaxone, respectively. The rate of ciprofloxacin and azithromycin resistance was $48.7 \%$ and $5.3 \%$, respectively. Two (0.1\%) isolates displayed high-level resistance to azithromycin, i.e. a minimum inhibitory concentration (MIC) $\geq 256$ $\mathrm{mg} / \mathrm{L}$. The current report further highlights the public health need to implement the European response plan, including further strengthening of Euro-GASP, to control and manage the threat of multidrug resistant N. gonorrhoeae.

\section{Introduction}

Neisseria gonorrhoeae has consistently developed resistance to antimicrobials used therapeutically for gonorrhoea, including penicillins, macrolides, tetracyclines and fluoroquinolones [1,2]. Surveillance of $N$. gonorrhoeae antimicrobial resistance is essential to monitor the emergence and spread of the resistance and to inform treatment guidelines. Furthermore, surveillance of antimicrobial resistance as well as treatment failures is also crucial, as reports are emerging of decreased susceptibility, in vitro resistance and clinical failure of the last line of agents for antimicrobial monotherapy: the extended-spectrum cephalosporins, cefixime (oral) and ceftriaxone (injectable) [2-6]. The European management guidelines [7] have recently been revised to recommend ceftriaxone (500 $\mathrm{mg}$ intramuscularly) in combination with azithromycin (2 g single oral dose) for first-line treatment of all uncomplicated gonorrhoea cases, in response to the emerging in vitro and in vivo resistance to cefixime and ceftriaxone.

Since 2009 the European gonococcal antimicrobial surveillance programme (Euro-GASP) has been implemented by the European Centre for Disease Prevention and Control (ECDC) as a sentinel surveillance system across Member States of the European Union (EU) and European Economic Area (EEA) to monitor antimicrobial susceptibility in N. gonorrhoeae [8]. Here we describe the spread of gonococcal isolates with in vitro resistance to cefixime and resistance to other antimicrobials surveyed across Europe, and the subsequent European response [9] to the threat of multidrug-resistant $N$. gonorrhoeae (MDR-NG) [10].

\section{Methods}

During 2011 N. gonorrhoeae isolates were collected from 21 participating countries (Table 1) and examined during two periods: May/June and November/ December. Participating countries followed one of two paths. There was a centralised testing model [11], in which antimicrobial susceptibility testing was performed on all isolates centrally by Etest (bioMérieux, Marcy l'Etoile, France) to determine the minimum inhibitory concentration (MIC) of cefixime and ceftriaxone or agar dilution for ciprofloxacin, azithromycin, spectinomycin and gentamicin. Alternatively, decentralised testing was performed, i.e. antimicrobial susceptibility testing was performed in the participant's own national reference or local laboratory. In 2011, ten countries performed decentralised antimicrobial susceptibility testing, by Etest or agar dilution (Table 1). As well as countries fulfilling the criteria for decentralised testing [11], an external quality assessment programme and a panel of control strains [11] were established to ensure comparability of data in this hybrid testing model.

The statistical significance of any changes in the proportion of isolates with resistance to tested antimicrobials 
Resistance to cefixime, ciprofloxacin and azithromycin in Neisseria gonorrhoeae isolates from 21 European Union/European Economic Area countries, 2011

\begin{tabular}{|c|c|c|c|c|c|c|c|c|}
\hline \multirow{3}{*}{ Country } & \multirow{3}{*}{$\begin{array}{c}\text { Number of } \\
\text { isolates tested }\end{array}$} & \multicolumn{6}{|c|}{ Resistance } & \multirow{3}{*}{ Method of testing } \\
\hline & & \multicolumn{2}{|c|}{ Cefixime } & \multicolumn{2}{|c|}{ Ciprofloxacin } & \multicolumn{2}{|c|}{ Azithromycin } & \\
\hline & & Number & $\%$ & Number & $\%$ & Number & $\%$ & \\
\hline Austria & 106 & 14 & 13.2 & 72 & 67.9 & 13 & 12.3 & Centralised \\
\hline Belgium & 110 & 1 & 0.9 & 61 & 55.5 & 4 & 3.6 & Decentralised (AG) \\
\hline Cyprus & 10 & 1 & 10.0 & 8 & 80.0 & 1 & 10.0 & Centralised \\
\hline Denmark & 125 & 25 & 20 & 73 & 58.4 & 15 & 12.0 & Decentralised (Etest) \\
\hline France & 109 & 0 & 0.0 & 49 & 45.0 & 2 & 1.8 & Decentralised (Etest) \\
\hline Germany & 108 & 11 & 10.2 & 55 & 50.9 & 1 & 0.9 & Centralised \\
\hline Greece & 100 & 3 & 3.0 & 74 & 74.0 & 5 & $7.9^{\mathrm{a}}$ & Decentralised (Etest) \\
\hline Hungary & 13 & 1 & 7.7 & 8 & 61.5 & 0 & 0.0 & Centralised \\
\hline Ireland & 64 & 2 & 3.1 & 9 & 14.1 & 5 & 7.8 & Centralised \\
\hline Italy & 99 & 3 & 3.0 & 60 & 60.6 & 4 & 4.0 & Decentralised (Etest) \\
\hline Latvia & 28 & 0 & 0.0 & 8 & 28.6 & 1 & 3.6 & Centralised \\
\hline Malta & 13 & 1 & 7.7 & 9 & 69.2 & 0 & 0.0 & Centralised \\
\hline Netherlands & 217 & 0 & 0.0 & 56 & 25.8 & 12 & 5.5 & Decentralised (Etest) \\
\hline Norway & 77 & 1 & 1.3 & 25 & 32.5 & 3 & 3.9 & Centralised \\
\hline Portugal & 109 & 0 & 0.0 & 46 & $46.5^{b}$ & 8 & 7.3 & Decentralised (Etest) \\
\hline Romania & 26 & 4 & 15.4 & 21 & 80.8 & 2 & 7.7 & Centralised \\
\hline Slovakia & 113 & 41 & 36.3 & 80 & 70.8 & 7 & 6.2 & Centralised \\
\hline Slovenia & 19 & 7 & 36.8 & 14 & 73.7 & 1 & 5.3 & Centralised \\
\hline Spain & 100 & 15 & 15.0 & 59 & 59.0 & 14 & 14.0 & Decentralised (AG) \\
\hline Sweden & 105 & 8 & 7.6 & 60 & 57.1 & 1 & 1.0 & Decentralised (Etest) \\
\hline United Kingdom & 251 & 7 & 2.8 & 75 & 29.9 & 0 & 0.0 & Decentralised (AG) \\
\hline Total & $\begin{array}{c}\text { Total: } 1,902 \\
\text { (ciprofloxacin } \\
\text { total 1,892, } \\
\text { azithromycin } \\
\text { total 1,865) } \\
\end{array}$ & 145 & 7.6 & 922 & 48.7 & 99 & $5 \cdot 3$ & \\
\hline
\end{tabular}

AG: agar dilution.

a Calculated from 63 isolates with azithromycin results.

b Calculated from 99 isolates with ciprofloxacin results.

between years was determined by the Z-test (chosen due to large sample size and dichotomous variables).

\section{Results}

A total of 1,902 N. gonorrhoeae isolates from 21 participating countries were examined in 2011, representing an increase from the 1,766 and 1,366 isolates received from 17 countries in 2010 and 2009, respectively.

The proportion of isolates that displayed in vitro resistance (formerly described as decreased susceptibility) [11] to cefixime was $7.6 \%(145 / 1,902$, Tables 1 and 2$)$ in 2011 using the European Committee on Antimicrobial Susceptibility Testing (EUCAST) breakpoint (MIC>0.12 $\mathrm{mg} / \mathrm{L})[12]$. This is a minor decrease compared to 2010 $(8.7 \%$ versus $7.6 \%$, Z-test, $p=0.25)$ but still significantly higher than in 2009 (5.1\% versus $7.6 \%$, Z-test, $\mathrm{p}=0.005$ ) (Table 2). In 2010 and 2011, cefixime resistant isolates were also detected in 17 countries, compared to only 10 in 2009 (Figure). Seventeen isolates had an MIC of $0.5 \mathrm{mg} / \mathrm{L}$ in 2011, which is an increase from two and four isolates in 2010 and 2009, respectively. All isolates showing in vitro resistance to cefixime were additionally resistant to ciprofloxacin (MIC>0.5 mg/L). In 2011, the first 10 (0.5\%) Euro-GASP isolates with in vitro resistance to ceftriaxone $(>0.12 \mathrm{mg} / \mathrm{L})$ were identified from the same geographical area (Austria and Germany). All ten isolates had MICs of cefixime of at least $0.12 \mathrm{mg} / \mathrm{L}$ and were also resistant to ciprofloxacin.

The rate of ciprofloxacin resistance decreased significantly from $62.7 \%(857 / 1,366)$ in 2009 to $48.7 \%$ $(922 / 1,892)$ in 2011 (Z-test, p<0.0002), and a significant decrease from $13.2 \%(180 / 1,366)$ to $5.3 \%(99 / 1,865)$ was observed for azithromycin resistance (MIC>0.5 $\mathrm{mg} / \mathrm{L}$; Z-test, p<0.0002) (Table 2), including two (0.1\%) isolates displaying high-level resistance to azithromy$\operatorname{cin}(\mathrm{MIC} \geq 256 \mathrm{mg} / \mathrm{L})$ in 2011 .

The modal MIC to gentamicin was $8 \mathrm{mg} / \mathrm{L}$ (MIC range: 0.5 to $16 \mathrm{mg} / \mathrm{L}$ ) and no resistance to spectinomycin (MIC range: 1.5 to $64 \mathrm{mg} / \mathrm{L}$ ) was demonstrated. 
Overall proportion of Neisseria gonorrhoeae isolates from 21 European Union/European Economic Area countries with resistance to cefixime, ciprofloxacin and azithromycin, 2009-2011

\begin{tabular}{|l|c|c|c|c|c|c|c|c|c|}
\hline & \multicolumn{3}{|c}{ Cefixime resistant } & \multicolumn{2}{c|}{ Ciprofloxacin resistant } & \multicolumn{3}{c|}{ Azithromycin resistant } \\
\cline { 2 - 11 } & $\begin{array}{c}\text { Cefixime } \\
\text { resistant/ } \\
\text { total }\end{array}$ & Percentage & $\begin{array}{c}95 \% \\
\text { confidence } \\
\text { intervals }\end{array}$ & $\begin{array}{c}\text { Ciprofloxacin } \\
\text { resistant/total }\end{array}$ & Percentage & $\begin{array}{c}95 \% \\
\text { confidence } \\
\text { intervals }\end{array}$ & $\begin{array}{c}\text { Azithromycin } \\
\text { resistant/total }\end{array}$ & $\begin{array}{c}\text { Percentage } \\
\text { confidence } \\
\text { intervals }\end{array}$ \\
\hline 2009 & $70 / 1,366$ & 5.1 & $4.01-6.4$ & $857 / 1,366$ & 62.7 & $60.2-65.3$ & $180 / 1,366$ & 13.2 & $11.4-15$ \\
\hline 2010 & $153 / 1,766$ & 8.7 & $7.4-10.1$ & $930 / 1,766$ & 52.7 & $50.3-55$ & $127 / 1,766$ & 7.2 & $6.02-8.5$ \\
\hline 2011 & $145 / 1,902$ & 7.6 & $6.5-8.9$ & $922 / 1,892$ & 48.7 & $46.5-51$ & $99 / 1,865$ & 5.3 & $4.4-6.4$ \\
\hline
\end{tabular}

\section{Discussion}

The results from Euro-GASP have demonstrated emerging cefixime and ceftriaxone resistance across the $\mathrm{EU} /$ EAA region. The levels of in vitro resistance to cefixime and ceftriaxone using the EUCAST breakpoints were $7.6 \%$ and $0.5 \%$, respectively, in 2011 . For comparison, the level of in vitro resistance to cefixime was $5.1 \%$ and $8.7 \%$ in 2009 and 2010 , respectively, and no isolates with in vitro resistance to ceftriaxone have been identified before 2011. However it should be noted that definitive breakpoints have not yet been established for cefixime and ceftriaxone, and several treatment failures with cefixime $(200 \mathrm{mg} \times 2)$ [13] and cefixime $400 \mathrm{mg}$ [6] have previously been caused by isolates with lower cefixime MICs than the tentative EUCAST breakpoint (>0.12 mg/L). Using an MIC breakpoint of $>0.06 \mathrm{mg} / \mathrm{L}$ for cefixime resulted in much higher proportions of the Euro-GASP isolates, $22.7 \%(400 / 1,766)$ and $18.6 \%(353 / 1,902)$, displaying in vitro resistance to cefixime in 2010 and 2011 respectively. And again the same breakpoint applied to ceftriaxone gave more isolates displaying in vitro resistance: $1.4 \%$ (24/1,699, 2010) and 2.5\% $(47 / 1,902,2011)$.

Even though the levels of resistance to ciprofloxacin and azithromycin decreased significantly, the resistance level is still too high for these antimicrobials to be used for empirical antimicrobial monotherapy [14], unless the susceptibility has been confirmed with antimicrobial susceptibility testing before initiating the therapy of the individual gonorrhoea cases. The wide variation in resistance rates across the different countries (e.g. $0-36.8 \%$ for cefixime in vitro resistance) represents the few isolates from some countries and the very diverse region covered by Euro-GASP.

Both gentamicin and spectinomycin are potential options for gonorrhoea treatment; however the lack of sufficient clinical efficacy and safety data and breakpoints for gentamicin $[2,4,15,16]$, as well as the difficulties in acquiring spectinomycin in most countries, the fear of rapidly selected resistance and the reduced

\section{FIGURE}

Geographical distribution of gonococcal isolates with in vitro resistance to cefixime (>0.12 mg/L) in Euro-GASP participating countries, 2009-2011

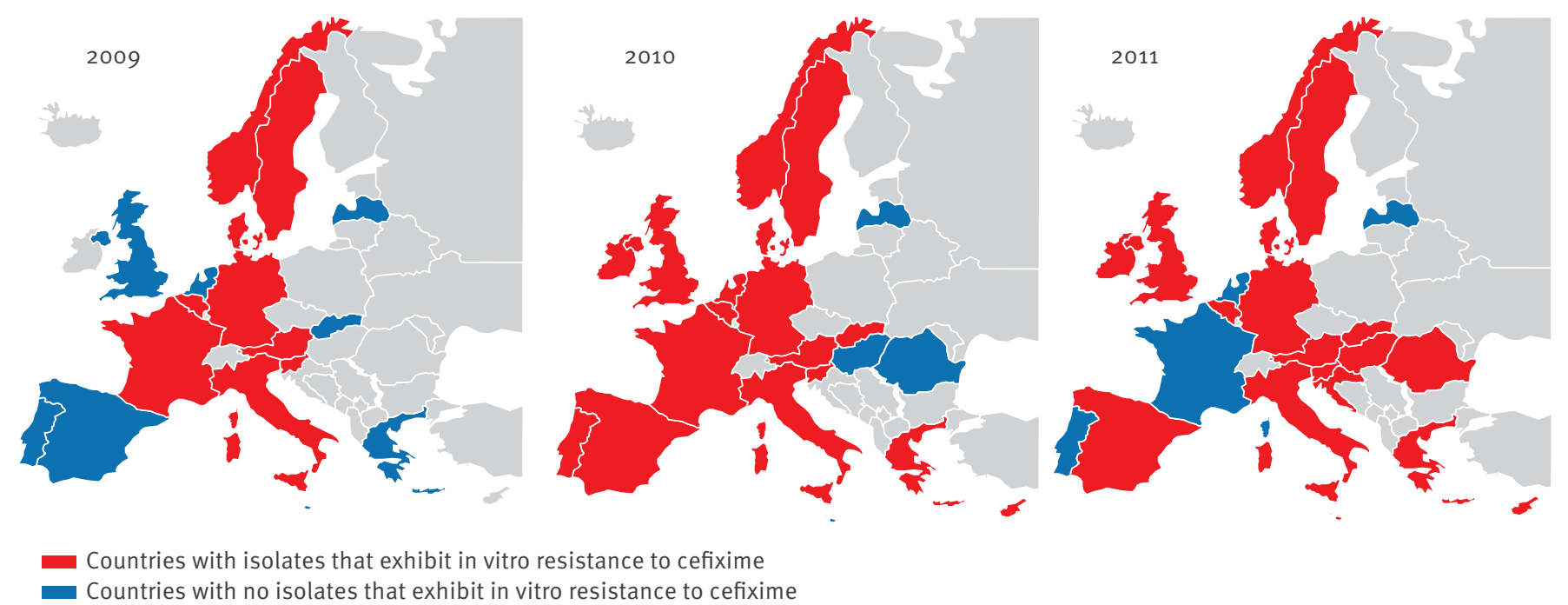


effectiveness of spectinomycin at clearing pharyngeal infections $[2,4,17]$ make these options less than satisfactory for first-line antimicrobial monotherapy. Our results clearly show that new, or combinations of current, antimicrobials are desperately needed to maintain gonorrhoea as a treatable disease, and that every effort must be made to preserve the efficacy of existing therapeutic options.

The decreasing susceptibility to the extended-spectrum cephalosporins, the increasing number of reported treatment failures to extended-spectrum cephalosporins (particularly cefixime), the associated morbidity with $N$. gonorrhoeae infection and lack of alternative treatment options have led to the development of a response plan to control and manage the threat of MDR-NG in Europe and to support Member States in EU and EEA in their national responses to MDR-NG [3,9]. Euro-GASP is a sentinel surveillance system and so is unable to detect treatment failures; the response plan [9] therefore includes a strategy for the detection and verification of treatment failures in a timely manner. Molecular tests to diagnose gonorrhoea are advantageous in that they are highly sensitive and rapid, are amenable to high-throughput and do not require an invasive specimen. However the European response plan [9] strongly emphasises that continued use of culture in sentinel sites is key to obtaining information on antimicrobial susceptibility of $N$. gonorrhoeae isolates, which is essential in order to detect emerging resistance. Even though an increase in the number of participating countries and progress in obtaining isolates for Euro-GASP in some countries have contributed to the increase in isolate numbers over the years, an absence of participation and low isolate numbers from some countries, along with differences in representativeness are limitations of Euro-GASP. Therefore as part of the European response, Euro-GASP will be strengthened to ensure a greater representation of $N$. gonorrhoeae antimicrobial resistance profiles and associated epidemiological information in Europe. Training will be provided to enable capacity building and encourage the collection of isolates from countries where no susceptibility testing is currently performed. Decentralised testing will be further promoted to improve timeliness of reporting, engage national stakeholders and facilitate the sustainability of Euro-GASP. Finally, the awareness of policy makers, clinicians, patients, and key populations will be enhanced [9]. The European response [9] aims to implement the actions as specified within the World Health Organization global action plan on antimicrobial resistance of $N$. gonorrhoeae [18].

Effective control of gonorrhoea relies entirely on appropriate treatment with antibiotics, along with effective prevention, timely diagnostics, contact tracing (including diagnostics, treatment and notification of contacts), and surveillance. Both the European response plan [9] and the revised European management guidelines [7] contribute to the fight to keep gonorrhoea a treatable infection. A further benefit of the European response
[9] is that a multidisciplinary collaboration between national and international stakeholders is developed, a network that will be valuable also for future gonococcal challenges.

\section{Authors' contributions}

M Cole analysed the data and drafted the manuscript. M van de Laar and G Spiteri coordinated Euro-GASP from ECDC. The laboratories of $C$ Ison, S Chisholm, S Hoffmann and M Unemo contributed isolate data for the study. All authors contributed to the manuscript.

\section{Acknowledgements}

We acknowledge the European STI surveillance network for their contribution in the development and implementation of Euro-GASP and the submission of gonococcal isolates and data. We further wish to thank Nerteley Quaye, Lene Berthelsen, Ronza Hadad and Emma Johansson for performing the laboratory work, and Katy Town for the statistical analysis.

The study was funded by the European Centre for Disease Prevention and Control (Framework Contract No. $\mathrm{ECDC} / 09 / 015)$.

Some of the results described in this manuscript have previously been published by ECDC at http://www.ecdc.europa. eu/en/publications/publications/gonococcal-antimicrobialsusceptibility-surveillance-27-mar-2013.pdf.

\section{References}

1. Lewis DA. The Gonococcus fights back: is this time a knock out? Sex Transm Infect. 2010;86(6):415-21. http://dx.doi. org/10.1136/sti.2010.042648

2. Unemo M, Shafer WM. Antimicrobial resistance in Neisseria gonorrhoeae in the 21st Century: past, evolution, and future. Clin Microbiol Rev. 2014;27(3):587-613. http://dx.doi. org/10.1128/CMR.00010-14

3. Van de Laar M, Spiteri G. Increasing trends of gonorrhoea and syphilis and the threat of drug-resistant gonorrhoea in Europe. Euro Surveill. 2012;17(29): pii=20225.

4. Unemo M, Nicholas RA. Emergence of multidrug-resistant, extensively drug-resistant and untreatable gonorrhea. Future Microbiol. 2012;7(12):1401-22. http://dx.doi.org/10.2217/ $\mathrm{fmb} .12 .117$

5. Lewis DA, Sriruttan C, Muller EE, Golparian D, Gumede L, Fick D, et al. Phenotypic and genetic characterization of the first two cases of extended-spectrum-cephalosporinresistant Neisseria gonorrhoeae infection in South Africa and association with cefixime treatment failure. J Antimicrob Chemother. 2013;68(6):1267-70. http://dx.doi.org/10.1093/jac/ dkto34

6. Allen VG, Mitterni L, Seah C, Rebbapragada A, Martin IE, Lee C, et al. Neisseria gonorrhoeae treatment failure and susceptibility to cefixime in Toronto, Canada. JAMA. 2013;309(2):163-70. http://dx.doi.org/10.1001/ jama.2012.176575

7. Bignell C, Unemo M, on behalf of the European STI Guidelines Editorial Board. 2012 European guideline on the diagnosis and treatment of gonorrhoea in adults. Int J STD AIDS. 2013;24(2):85-92. http://dx.doi. org/10.1177/0956462412472837

8. Cole MJ, Unemo M, Hoffmann S, Chisholm SA, Ison CA, van de Laar MJ. The European gonococcal antimicrobial surveillance programme, 2009. Euro Surveill. 2011;16(42): pii=19995.

9. European Centre for Disease Prevention and Control (ECDC). Response plan to control and manage the threat of multidrugresistant gonorrhoea in Europe. Stockholm: ECDC; 2012. Available from: http://ecdc.europa.eu/en/publications/ Publications/1206-ECDC-MDR-gonorrhoea-response-plan.pdf 
10. Tapsall JW, Ndowa F, Lewis DA, Unemo M. Meeting the public health challenge of multidrug- and extensively drugresistant Neisseria gonorrhoeae. Expert Rev Anti Infect Ther. 2009;7(7):821-34. http://dx.doi.org/10.1586/eri.09.63

11. European Centre for Disease Prevention and Control (ECDC). Gonococcal antimicrobial susceptibility surveillance in Europe 2011. Stockholm: ECDC; 2013. Available from: http://www. ecdc.europa.eu/en/publications/publications/gonococcalantimicrobial-susceptibility-surveillance-27-mar-2013.pdf

12. European Committee on Antimicrobial Susceptibility Testing (EUCAST). Breakpoint tables for interpretation of MICs and zone diameters. Växjö : EUCAST; 2012. Available from: http:// www.eucast.org/fileadmin/src/media/PDFs/EUCAST_files/ Breakpoint_tables/Breakpoint_table_v_2.0_120221.pdf

13. Deguchi T, Yasuda M, Yokoi S, Ishida K, Ito M, Ishihara S, et al. Treatment of uncomplicated gonococcal urethritis by doubledosing of $200 \mathrm{mg}$ cefixime at a 6-h interval. J Infect Chemother 2003;9(1):35-9. http://dx.doi.org/10.1007/s10156-002-0204-8

14. Tapsall J. Antimicrobial resistance in Neisseria gonorrhoeae. Geneva: World Health Organization; 2001. Available from: http://www.who.int/drugresistance/Antimicrobial_resistance in_Neisseria_gonorrhoeae.pdf

15. Dowell D, Kirkcaldy RD. Effectiveness of gentamicin for gonorrhoea treatment: systematic review and meta-analysis. Sex Transm Infect. 2012;88(8):589-94. http://dx.doi. org/10.1136/sextrans-2012-050604

16. Chisholm SA, Quaye N, Cole MJ, Fredlund H, Hoffmann S, Jensen JS, et al. An evaluation of gentamicin susceptibility of Neisseria gonorrhoeae isolates in Europe. J Antimicrob Chemother. 2011;66(3):592-5. http://dx.doi.org/10.1093/jac/ dkq476

17. Moran JS. Treating uncomplicated Neisseria gonorrhoeae infections: is the anatomic site of infection important? Sex Transm Dis. 1995;22(1):39-47. http://dx.doi. org/10.1097/00007435-199501000-00007

18. Ndowa F, Lusti-Narasimhan M, Unemo $M$. The serious threat of multidrug-resistant and untreatable gonorrhoea: the pressing need for global action to control the spread of antimicrobial resistance, and mitigate the impact on sexual and reproductive health. Sex Transm Infect. 2012;88(5):317-8. http://dx.doi. org/10.1136/sextrans-2012-050674 